\title{
An algorithm for toroidal field harmonics computation in arbitrary magnetic configurations
}

\author{
L. Gambini, M. Breschi, E. Felcini, A. Cristofolini, L. Bottura
}

\begin{abstract}
Toroidal magnetic configurations are widely exploited in industry and scientific research, involving a vast spectrum of applications, such as thermonuclear fusion, particle detectors, SMES systems and medical devices. To properly design and analyse these systems, it is crucial to determine the magnetic field generated by different configurations. The multipole expansion theory can be applied to the analysis of toroidal configurations, by solving the Laplace equation for the magnetic scalar potential in toroidal coordinates. Contrarily to the case of accelerator magnets with straight axis, in this case the correlation between the current distribution and the field harmonics cannot easily be identified. This paper proposes a methodology for the computation of field harmonics in toroidal coordinates, which is validated by comparison with the results obtained through the Biot-Savart law. This work was carried out in the frame of the GaToroid project ongoing at CERN.
\end{abstract}

Index Terms - Curve fitting, Harmonic analysis, Laplace equation, Toroidal magnetic fields

\section{INTRODUCTION}

$\mathbf{T}$ OROIDAL magnets are widely exploited in industry and scientific research, involving a vast spectrum of applications, such as thermonuclear fusion, particle detectors, SMES systems and medical devices. Toroidal configurations may involve different number of coils of different planar and three-dimensional geometries; to properly analyze these systems, it is crucial to determine the magnetic field generated by the various configurations.

The multipole expansion theory in cylindrical coordinates is widely adopted to describe the magnetic field of particle accelerator magnets with straight axis [1]. The main advantage of this analytical description is the possibility of identifying multipolar field components of the magnetic system and use them to predict the interaction of a charged particle beam with the field itself. In this case, the correlation between current distribution and field harmonics is well known.

The multipole expansion theory can also be applied to the analysis of toroidal configurations, by solving the Laplace equation for the magnetic scalar potential in toroidal coordinates [2]-[5]. In this case however, the correlation between the current distribution and the field harmonics cannot easily be identified.

This paper proposes a methodology for the determination of the field harmonics in toroidal coordinates, based on the work

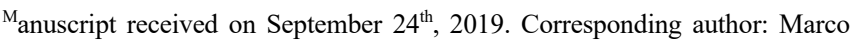
Breschi.

M. Breschi, A. Cristofolini, and L. Gambini are with the Department of Electrical, Electronic, and Information Engineering at the University of Bologna, Italy (e-mail: marco.breschi@unibo.it).
}

introduced in [6]. The starting point of the model is the calculation of the magnetic scalar potential, based on a hybrid analytical-numerical approach, which was validated versus well-established software for electromagnetic calculations.

Several previous studies concerning toroidal field harmonics propose simplified versions of the equations involved. Sometimes the expansion functions are normalized by means of halfinteger Legendre functions computed at a selected value of the radial toroidal coordinate for numerical purposes [2], [7]. The methodology proposed in this paper does not require any simplification, and allows one describing magnetic fields with satisfactory accuracy by using a rather low number of toroidal harmonics (less than 100 in the considered configurations).

\section{Multipole ExPAnsion In TOROIDAL COORDINATES}

The toroidal coordinate system $(\xi, \eta, \phi)$ is a set of three-dimensional orthogonal coordinates obtained by rotating the twodimensional bipolar coordinates $(\xi, \eta)$ around the axis which separates the two foci [8], shown in Fig. 1. Strictly speaking, ( $\xi$, 7) can be associated to the radius and the poloidal angle of a more intuitive cylindrical coordinate system.

The procedure followed for the evaluation of the field harmonics is based on the Laplace's equation solution for the magnetic scalar potential, in a suitable coordinate system [1].

The inner region of a toroidal system is in vacuum and no current lines are present; in this area the magnetic field is irrotational, hence it can be expressed as the gradient of the scalar potential $\boldsymbol{H}=-\nabla \psi$, provided that the toroidal region is reduced to a simply connected domain. Furthermore, the magnetic field $\boldsymbol{H}$ is solenoidal, consequently we can write the Laplace's equation for the scalar potential $\nabla^{2} \psi=0$ [9]. The Laplace's equation can be solved in toroidal coordinates employing the R-separation technique [10]. Since the toroidal geometry represents a multiply connected domain, it is necessary to add to the general solution reported in [10] the term $M_{00}^{\phi} \phi$ (see (1)), which allows fulfilling the Ampere's law. This term, called ideal contribution, corresponds to the magnetic field generated by a homogenous current distribution on a toroidal surface. This contribution does not depend on the coil geometry; it can be demonstrated that $M_{00}^{\phi}=N I / 2 \pi$, where $N$ is the number of coils of the magnetic configuration and $I$ the current flowing in each coil [2]. Assuming that no current is flowing inside the torus, $\xi$ takes

L. Bottura and E. Felcini are with the Magnets, Cryostats and Superconductors group at CERN, Geneva, Switzerland (e-mail: enrico.felcini@cern.ch). 
finite values in this region, and periodicity exists along $\phi$ and $\eta$, then the scalar potential can be written for the inner region of the torus as follows:

$$
\begin{aligned}
\psi(\xi, \eta, \phi)= & M_{00}^{\phi} \phi+\sqrt{\cosh (\xi)-\cos (\eta)} \sum_{m=0}^{M} \sum_{n=0}^{N} Q_{m-\frac{1}{2}}^{n}(\cosh (\xi)) \\
\times & {\left[M_{m, n}^{c c} \cos (n \phi) \cos (m \eta)+M_{m, n}^{c s} \cos (n \phi) \sin (m \eta)\right.} \\
& \left.+M_{m, n}^{s c} \sin (n \phi) \cos (m \eta)+M_{m, n}^{s s} \sin (n \phi) \sin (m \eta)\right]
\end{aligned}
$$

where $M_{i j}$ are the multipolar coefficients related to the field harmonics, and the terms $P_{m-1 / 2}^{n}$ and $Q_{m-1 / 2}^{n}$ are the half-integer Legendre polynomials of the first and second kind [11], respectively.

The magnetic field components are deduced by applying the gradient to the whole expression of the scalar potential, in toroidal coordinates.

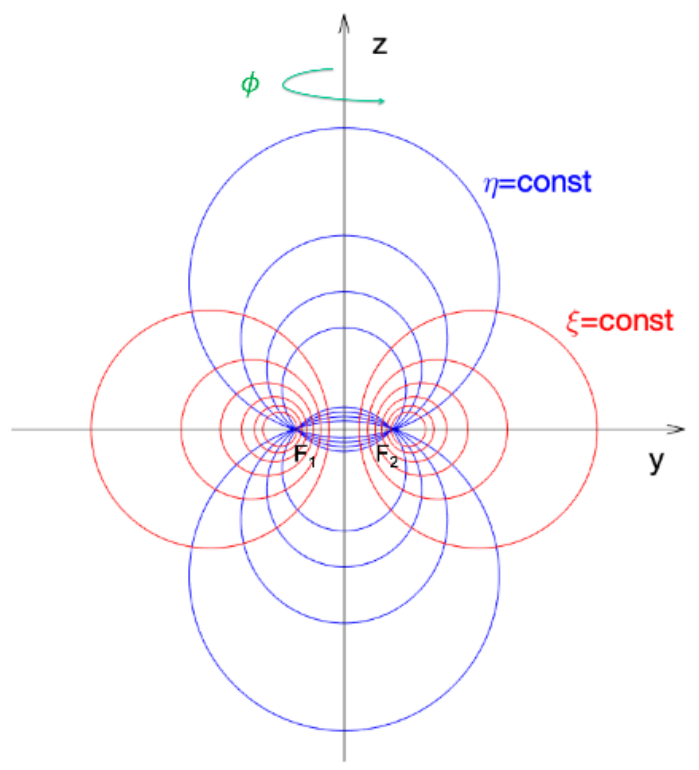

Fig. 1. Bipolar coordinate system. The rotation around the z-axis, quantified by the angle $\phi$, defines the toroidal coordinate system.

The expressions found are the following:

$$
\begin{array}{r}
H_{\xi}(\xi, \eta, \phi)=-\frac{k^{\frac{3}{2}}}{a} \sum_{m=0}^{M} \sum_{n=0}^{N}\left[M_{m, n}^{c c} \cos (n \phi) \cos (m \eta)+M_{m, n}^{c s} \cos (n \phi) \sin (m \eta)\right. \\
\left.+M_{m, n}^{s c} \sin (n \phi) \cos (m \eta)+M_{m, n}^{s s} \sin (n \phi) \sin (m \eta)\right] \\
\times\left[\frac{1}{2} k^{-1} \sinh (\xi) Q_{m-\frac{1}{2}}^{n}(\cosh (\xi))+\frac{\partial}{\partial \xi} Q_{m-\frac{1}{2}}^{n}(\cosh (\xi))\right]
\end{array}
$$

$$
\begin{array}{r}
H_{\eta}(\xi, \eta, \phi)=-\frac{k^{\frac{3}{2}}}{a} \sum_{m=0}^{M} \sum_{n=0}^{N} Q_{m-\frac{1}{2}}^{n}(\cosh (\xi))\left[\frac { 1 } { 2 } \operatorname { s i n } ( \eta ) k ^ { - 1 } \left(M_{m, n}^{c c} \cos (n \phi) \cos (m \eta)\right.\right. \\
\left.+M_{m, n}^{c s} \cos (n \phi) \sin (m \eta)+M_{m, n}^{s c} \sin (n \phi) \cos (m \eta)+M_{m, n}^{s s} \sin (n \phi) \sin (m \eta)\right) \\
+m\left(-M_{m, n}^{c c} \cos (n \phi) \sin (m \eta)+M_{m, n}^{c s} \cos (n \phi) \cos (m \eta)\right. \\
\left.\left.-M_{m, n}^{s c} \sin (n \phi) \sin (m \eta)+M_{m, n}^{s s} \sin (n \phi) \cos (m \eta)\right)\right]
\end{array}
$$

$$
\begin{aligned}
H_{\phi}(\xi, \eta, \phi)= & -\frac{k M_{00}^{\phi}}{a \sinh (\xi)}-\frac{k^{\frac{3}{2}}}{a \sinh (\xi)} \sum_{m=0}^{M} \sum_{n=0}^{N} n Q_{m-\frac{1}{2}}^{n}(\cosh (\xi)) \\
\times & {\left[-M_{m, n}^{c c} \sin (n \phi) \cos (m \eta)-M_{m, n}^{c s} \sin (n \phi) \sin (m \eta)\right.} \\
& \left.+M_{m, n}^{s c} \cos (n \phi) \cos (m \eta)+M_{m, n}^{s s} \cos (n \phi) \sin (m \eta)\right]
\end{aligned}
$$

where $k=\sqrt{\cosh (\xi)-\cos (\eta)}$. Once the coefficients of the multipolar expansion of the magnetic scalar potential are known, the magnetic field components can be computed with the equations (2) - (4).

\section{Methods AND PRocedures}

The methodology proposed here for the computation of the multipolar coefficients is based on two subsequent fitting procedures, where the coefficients are determined by non-linear least squares method. No simplifications of the expansion (1) are needed with this approach. The requirement for the application of this algorithm is the knowledge of the magnetic scalar potential at a fixed radial coordinate $\xi=\xi_{0}$, for $L$ values of the poloidal angle $\eta$ and $S$ values of the toroidal angle $\phi$. This set of points defines a reference grid. It has been verified that values of $L=S=200$ are a good compromise between accuracy of results and computational burden. The reference grid is defined by equally spaced points, although this is not a requirement for the use of the proposed method. For this work, the scalar potential has been computed with a procedure based on the evaluation of the solid angles subtending each current loop [12], employing the plane triangles approximation [13]. It is worth recalling that the scalar potential presents a discontinuity on an arbitrary surface bounded by the current loop. Still, its gradient (i.e. the magnetic field) remains a continuous function within the toroidal domain inside the coils [12].

The procedure described below is applied to the scalar potential $\psi^{*}=\psi-M_{00}^{\phi} \phi$, where the ideal contribution, known a priori, is removed to further ameliorate the computational accuracy. The first part of the procedure requires the magnetic scalar potential at a fixed value $\xi=\xi_{0}$ and at given angles $\eta=\eta l$, with $l=0,1, \ldots, L-1$. Therefore, $\psi^{*}$ is reduced to a function of $\phi$ only and the expression of the scalar potential can be written as:

$\psi^{*}\left(\xi_{0}, \eta_{l}, \phi\right)=\sum_{n=0}^{N}\left[C_{n}\left(\xi_{0}, \eta_{l}\right) \cos (n \phi)+D_{n}\left(\xi_{0}, \eta_{l}\right) \sin (n \phi)\right]$

where

$$
\begin{array}{r}
C_{n}\left(\xi_{0}, \eta_{l}\right)=\sqrt{\cosh \left(\xi_{0}\right)-\cos \left(\eta_{l}\right)} \sum_{m=0}^{M} Q_{m-\frac{1}{2}}^{n}\left(\cosh \left(\xi_{0}\right)\right)\left[M_{m, n}^{c c} \cos \left(m \eta_{l}\right)\right. \\
\left.+M_{m, n}^{c s} \sin \left(m \eta_{l}\right)\right] \\
D_{n}\left(\xi_{0}, \eta_{l}\right)=\sqrt{\cosh \left(\xi_{0}\right)-\cos \left(\eta_{l}\right)} \sum_{m=0}^{M} Q_{m-\frac{1}{2}}^{n}\left(\cosh \left(\xi_{0}\right)\right)\left[M_{m, n}^{s c} \cos \left(m \eta_{l}\right)\right. \\
\left.+M_{m, n}^{s s} \sin \left(m \eta_{l}\right)\right]
\end{array}
$$


First of all, it is necessary to choose the values of $N$ and $M$ at which the summations can be truncated. The values of $C_{n}$ and $D_{n}$ can be found by applying a fitting procedure, named Fitting 1 , which takes as an input the values of the scalar potential at $\xi$ $=\xi_{0}, \eta=\eta_{l}$ for $S$ equidistant values of $\phi_{s}=2 \pi s / S$, with $s=0,1$, ..., $S-1$. Equation (5) is used as a fitting equation in order to obtain the $2 N$ values of the coefficients $C_{n}\left(\xi_{0}, \eta_{l}\right)$ and $D_{n}\left(\xi_{0}, \eta_{l}\right)$.

The actual values of the multipolar components are undetermined at this stage. Indeed, the terms $C_{n}$ and $D_{n}$ involve many contributions of the toroidal harmonics, which need to be distinguished between each other.

To this purpose, it is necessary to apply all the steps described so far to each different value of $\eta l=2 \pi l / L$, with $l=0,1$, ..., $L-1$. The result of this last step is a set of $L$ values of $C_{n}$ and a set of $L$ values of $D_{n}$, which depend on the angle $\eta$ and on the fixed value $\xi_{0}$. These data can be interpreted as the known data of the previous fitting; therefore, it is possible to employ a similar method to fit them and obtain the coefficients $M_{c c}, M_{s c}$, $M_{c s}, M_{s s}$, for each value of $n$ and $m$. The two procedures implemented are referred to hereafter as Fitting $2 C$ and Fitting $2 D$ : the fitting equations are respectively (6) and (7). At the end of the fitting procedures, the values of each coefficient, introduced in the multipolar expansion of the scalar potential, are known.

Therefore, employing the described technique, the multipoles of a specific toroidal configuration can be identified. The multipolar coefficients can also be substituted in the expression of the magnetic field components (2), (3), (4).

\section{A. Validations}

The first method that can be used to validate the self-consistency of the proposed procedure consists in comparing the scalar potential computed with the solid angles method, referred to as $\psi_{s a}$, and the scalar potential obtained by inserting in (1) the multipolar coefficients found through the fitting procedure, indicated as $\psi_{m c}$. The study of the relative error between these two values of the scalar potential, computed with the ideal contribution included in the results, allows one verifying the correctness of the multipolar coefficients assessment.

The validity of the scalar potential computation and of its decomposition in multipolar coefficients can also be confirmed by replacing them in (2), (3) and (4) to determine the field components along the coordinate axes. These results can be directly compared with the magnetic field independently computed by means of the Biot-Savart law [14]. A further validation of the results was based on verifying that the computed magnetic field components respect the Ampere's law.

\section{RESUlts AND Discussion}

The aim of this section is to present some of the configurations which have been analyzed employing the methodology proposed in this paper. The input parameters characterizing the three studied configurations are summarized in Table 1. In order to demonstrate the absence of restrictions required by the algorithm, the three examples proposed here involve a different number of coils at the same angular coordinate and a non-circular coil shape; their geometry is shown in Fig. 2.

The simplest configuration analyzed consists of a toroid with 16 individual circular coils, equally distributed along the azimuthal coordinate, represented in blue in Fig. 2a). In the same figure, the red points represent the reference grid necessary for the implementation of the fitting procedure. Fig. 3 shows the comparison between the two scalar potentials $\psi_{s a}$ and $\psi_{m c}$, evaluated at a fixed $\eta=0.6 \mathrm{rad}$. The good agreement between these two potentials indicates the correctness of the identification procedure of the multipolar coefficients.

As for the validation by comparison with the independent Biot-Savart based field computation, the results obtained with the two methods for the component $H_{\phi}$ computed at fixed $\eta=$ $0.6 \mathrm{rad}$ are shown in Fig. 4. The maximum relative error found between the results of the two procedures is in the order of $10^{-5}$ $\%$.

A more complex configuration is the one represented in Fig. $2 b$ ), which involves coil grading with four non-concentric circular coils at the same toroidal section, as specified in Table I.
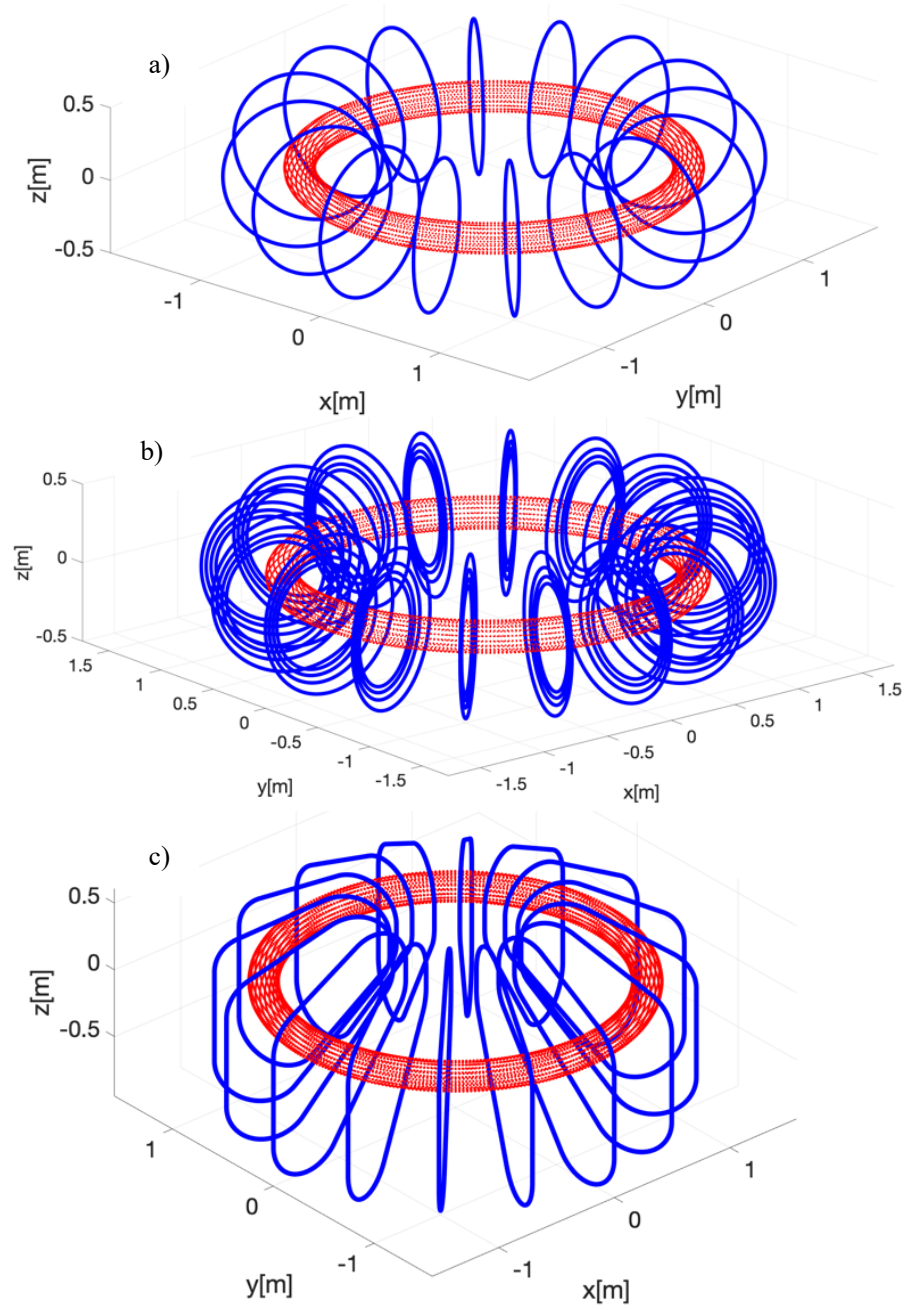

Fig. 2. Analyzed configurations: the blue points represent the coils, while the red points represent the reference grid. a) 16 individual circular coils. b) 16 graded circular coils, 4 coils in each section, 64 coils in total. c) 16 individual GaToroid coils. 
This type of configuration is applied to modify the typical $1 / r$ field dependence of the $H_{\phi}$ component. The procedure described in this work does not impose any restriction on the shape of the coils. For instance, it is possible to consider coils with the shape displayed in Fig. 2c), which have been proposed for the GaToroid system [15]-[17]. The relative errors found by comparing $\psi_{s a}$ and $\psi_{m c}$ for the three configurations is shown in Fig. 5, for three different choices of the upper limits for the computation of the infinite sums.

TABLE I

CONFIGURATIONS PARAMETERS

\begin{tabular}{cccc}
\hline \hline Parameter & Case A & Case B & Case C \\
\hline Number of coils & 16 & 16 & 16 \\
Number of coils per section & 1 & 4 & 1 \\
Current in each coil [A] & 1000 & 1000 & 1000 \\
Toroid major radius [m] & 1.25 & 1.33 & - \\
Toroid minor radius [m] & 0.5 & 0.33 & - \\
Reference grid major radius [m] & 1.25 & 1.25 & 1.25 \\
Reference grid minor radius [m] & 0.05 & 0.05 & 0.05 \\
\hline
\end{tabular}

In the case of graded coil, the third and the fourth parameters refer to the inner coil; these terms are not provided in the case of GaToroid coils, whose shape does not allow one to define radii.

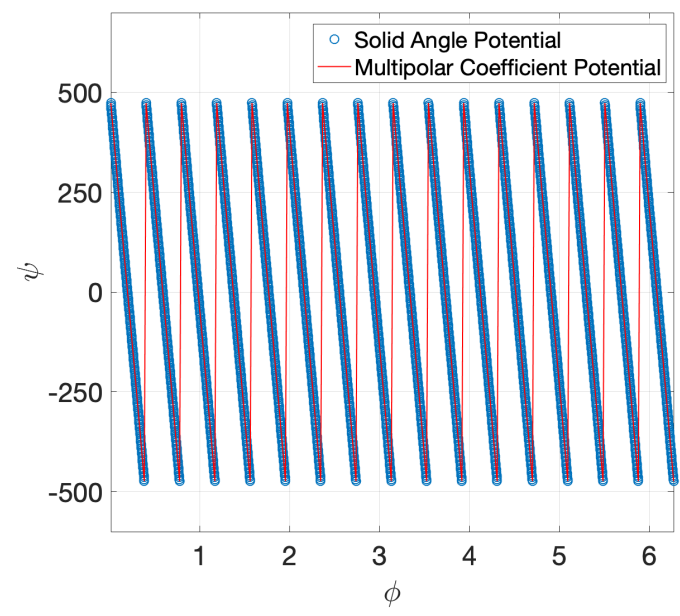

Fig. 3. Comparison between $\psi_{s a}$ and $\psi_{m c}$, for the Case a). For the sake of simplicity, the comparison is plotted at a fixed value of $\eta$, set to $0.6 \mathrm{rad}$.

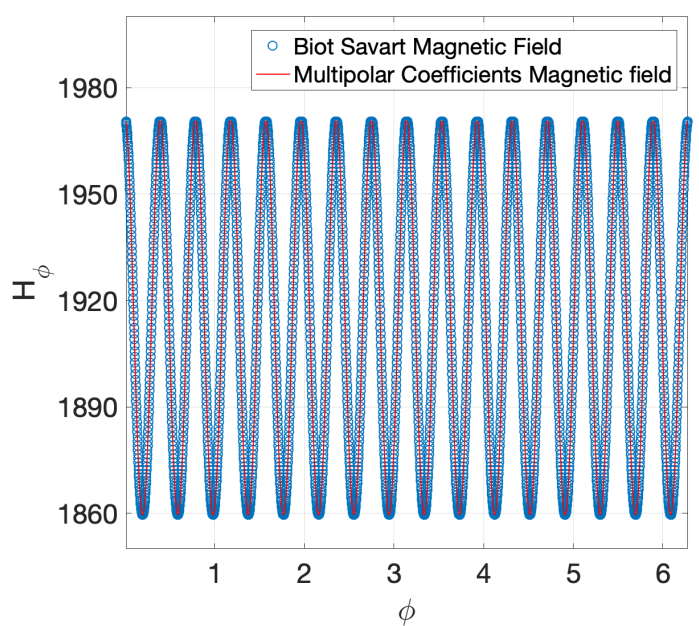

Fig. 4. Comparison between the magnetic field component $H_{\phi}$ computed with the Biot-Savart law and the same field component obtained by replacing the computed multipolar coefficients in (4), for Case a). For the sake of simplicity, the comparison is plotted at a fixed value of $\eta$, set to $0.6 \mathrm{rad}$.

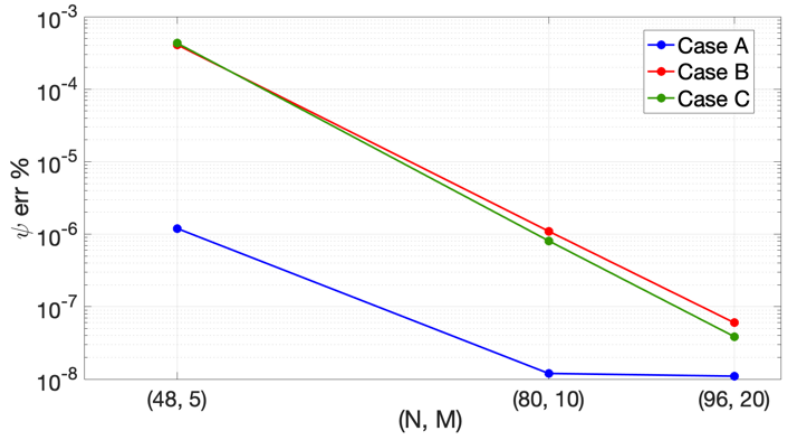

Fig. 5. Comparison of the relative error between the scalar potential obtained with the solid angles' method and the scalar potential found by replacing in (1) the computed multipolar coefficients, for the cases a), b) and c). Relative errors are evaluated for different ranges of the index $n=0: N$ and $m=0: M$.

In particular, the sums in $n$ and $m$ were truncated at $N(48$, $80,96)$ and $M(5,10,20)$ respectively.

Fig. 5 shows that a lower number of harmonics are required to achieve a correct reconstruction of the scalar potential in case a), given the simplicity and the symmetry of the magnetic configuration. In this case, choosing the index $n$ in the range 0:80 and the index $m$ in the range $0: 10$, the maximum relative error obtained from the comparison between $\psi_{s a}$ and $\psi_{m c}$ is $110^{-8} \%$.

As for cases b) and c), with $n=0: 96$ and $m=0: 20$, a relative error of $610^{-8} \%$ and $410^{-8} \%$ are obtained respectively.

Therefore, in order to reach the same accuracy, a higher number of harmonics is required for cases b) and c).

\section{CONCLUSION}

A novel methodology has been developed for the computation of field harmonics in toroidal coordinates, without any constraint on the number and shape of coils in the magnetic configuration. The self-consistency of the procedure was proved by reconstructing the given scalar potential by means of a finite sum of the toroidal multipolar components.

A further validation of the procedure was obtained by comparing the results on the magnetic field components with those found via an independent algorithm based on the Biot-Savart's law developed to this purpose.

The calculation accuracy obtained with the proposed methodology is well below the precision required for field quality assessment in particle accelerators, fusion machines and medical applications. The developed algorithm can be applied to explore the correlation between the shape and number of coils disposed along the torus and the generated multipolar components.

\section{ACKNOWLEDGMENT}

L. Brower from Lawrence Berkeley National Laboratory, US, B. van Milligen from CIEMAT, Madrid, Spain and S. Russenschuck from CERN, Geneva, Switzerland are gratefully acknowledged for fruitful discussions. 


\section{REFERENCES}

[1] S. Russenschuck, Field Computation for Accelerators Magnets. 2011.

[2] B. P. van Milligen and A. Lopez Fraguas, "Expansion of vacuum magnetic fields in toroidal harmonics," Comput. Phys. Commun., vol. 81, no. 1-2, pp. 74-90, 1994.

[3] L. Brouwer, S. Caspi, D. Robin, and W. Wan, "3D toroidal field multipoles for curved accelerator magnets," 2017.

[4] F. Alladio and F. Crisanti, "Analysis of MHD equilibria by toroidal multipolar expansions," Nucl. Fusion, vol. 26, no. 9, p. 1143, 1986.

[5] B. Faugeras, "Tokamak plasma boundary reconstruction using toroidal harmonics and an optimal control method," Fusion Sci. Technol., vol. 69, no. 2, pp. 495-504, 2016.

[6] L. Gambini, "Expansion of the magnetic flux density field in toroidal harmonics." Master thesis, CERN-THESIS-2018-040, Bologna U., Bologna, Italy, 2018.

[7] G.T. Bodwin, H.S. Chunga and J. Reponda, "Implementation of Maxwell's equations in the reconstruction of the magnetic field in the g-2 storage ring," JINST, Volume 14, July 2019.

[8] W. D. D'haeseleer, W. N. G. Hitchon, J. D. Callen, and J. L. Shohet, Flux Coordinates and Magnetic Field Structure: A Guide to a Fundamental Tool of Plasma Theory. Springer Berlin Heidelberg, 2012.

[9] S.Turner, "Measurement and Alignment of Accelerator and Detector Magnets," CAS - CERN Accelerator School, https://cds.cern.ch/record/318977/ 1997.

[10] P. Moon and D. E. Spencer, "Field theory handbook: including coordinate systems, differential equations and their solutions. Springer, 2012.

[11] M. Abramowitz and I. A. Stegun, Handbook of mathematical functions with formulas, graphs, and mathematical table. in US Department of Commerce, National Bureau of Standards Applied Mathematics series 55, 1965.

[12] J. V Stewart, Intermediate electromagnetic theory. World Scientific Publishing Company, 2001.

[13] A. Van Oosterom and J. Strackee, "The solid angle of a plane triangle," IEEE Trans. Biomed. Eng., no. 2, pp. 125-126, 1983.

[14] J. D. Jackson, Classical electrodynamics. AAPT, 1999.

[15] L. Bottura, "A Gantry and apparatus for focussing beams of charged particles," Patent WO2019/224215, 2019.

[16] E. Felcini, L. Bottura, J. Nugteren, G. de Rijk, G. Kirby, and B. Dutoit, "Magnetic Design of a Superconducting Toroidal Gantry for Hadron Therapy," IEEE Trans. Appl. Supercond., vol. PP, p. 1, Jan. 2020.

[17] L. Bottura and E. Felcini, "Novel Concept of a Gantry for Hadron Therapy Based on Steady Toroidal Magnets," Cern Intern. Report, Unpubl., To be Submitt. to Nucl. Instrum. Methods. 